\title{
In- and out-of-phase thermomechanical fatigue of a Ni-based single-crystal superalloy
}

\author{
Mikael Segersäll ${ }^{1, \text { a }}$, Johan J. Moverare ${ }^{1,2}$, Daniel Leidermark ${ }^{1}$, and Sten Johansson ${ }^{1}$ \\ ${ }^{1}$ Department of Management and Engineering, Linköping University, 58183 Linköping, Sweden \\ ${ }^{2}$ Siemens Industrial Turbomachinery AB, 16283 Finspång, Sweden
}

\begin{abstract}
In this study, the difference between in-phase (IP) and out-of-phase (OP) thermomechanical fatigue (TMF) cycling from 100 to $750^{\circ} \mathrm{C}$ has been investigated for the Ni-based single-crystal superalloy MD2. In addition, two different crystal orientations were studied, the $\langle 001\rangle$ and $\langle 011\rangle$ orientations respectively. When comparing IP and OP TMF lives, a strain range dependency is found for the $\langle 001\rangle$ direction. For high strain ranges, IP cycling leads to a higher number of cycles to failure compared to OP. However at lower strain ranges, OP cycling leads to a higher number of cycles to failure compared to IP. Microstructure investigation shows that for the $\langle 001\rangle$ direction, deformation twinning within the $\gamma / \gamma^{\prime}$-microstructure is much more pronounced during OP conditions compared to IP. However for the $\langle 011\rangle$ direction, the opposite is observed; twinning is more pronounced during IP TMF. From the microstructure investigation it is also visible that intersections between twins seems to trigger formation of TCP phases and recrystallization. These intersections also work as initiation points for TMF damage.
\end{abstract}

\section{Introduction}

Ni-based superalloys casted in single-crystal form are often used for first row turbine blades in gas turbines and aero engines. First row turbine blades are subjected to the highest temperatures (above $1000^{\circ} \mathrm{C}$ ) of all blades and at the same time, high centrifugal forces. Excellent fatigue and creep properties are of great importance for turbine blades, and both properties are enhanced by use of single-crystal instead of poly-crystal material [1]. Superalloys consist of the typical $\gamma / \gamma^{\prime}$-microstructure where the $\gamma$-matrix is surrounding precipitates of cuboidal $\gamma^{\prime}$-phase which has an $\mathrm{L1}_{2}$-ordered structure. Performing thermomechanical fatigue (TMF) testing in the laboratory is one way to investigate component near service conditions. Several types of cycles can be used during TMF testing. For example, an in-phase (IP) TMF cycle, will lead to creep relaxation in tension at maximum temperature and plastic deformation in compression at minimum temperature. This condition corresponds to cold spots on the turbine blade, which often are found close to cooling holes or inside cooling channels [2]. On the other hand, an out-of-phase (OP) TMF cycle, creep relaxation will occur in compression at maximum temperature and plastic deformation in tension at minimum temperature. OP TMF is more connected to hot spots on the turbine blade where the hot spots desire to expand by thermal expansion, but are prevented by their cooler surroundings. The hot spots are often found on the blade airfoil or

\footnotetext{
${ }^{a}$ Corresponding author: mikael.segersall@liu.se
}

platform. TMF damage on gas turbine blades is often more connected to hot spots, meaning that OP TMF is a more component near testing condition. Hence, studies concerning OP TMF are more common in the literature compared to IP TMF.

Han et al. [3] studied the difference between IP and OP TMF and found that OP fatigue lives were shorter than IP fatigue lives. This was explained by that the maximum tensile stress is obtained in the cold end of the OP TMF cycle. Other research has found that the difference between IP and OP TMF life seems to be strain range dependent [4]. In that study IP TMF life was found to be shorter than $\mathrm{OP}$ at high strain ranges. A better understanding regarding the deformation and damage mechanisms that occur during TMF is of great importance to study in order to understand what governs the TMF life. Previous studies $[5,6]$ have shown that the deformation within the $\gamma / \gamma^{\prime}$-microstructure is very localized and that twinning is a major deformation mechanism during OP TMF. Another study [7] showed that parallel twin plates grow on $\{111\}$ planes close to the crack tip and that those twins create a path for crack propagation. Kovarik et al. $[8,9]$ performed extensive research regarding the formation of microtwins during deformation of superalloys at intermediate temperatures $\left(660-700^{\circ} \mathrm{C}\right)$. It seems that the twinning partial dislocations propagate through the $\mathrm{L1}_{2}$-ordered $\gamma^{\prime}$-precipitates on $\{111\}$ planes. The rate limiting process of microtwinning seems to be re-ordering within the $\gamma^{\prime}$-phase.

The occurring deformation and damage mechanisms in superalloys during TMF conditions are not yet fully understood and have to be further investigated. In this 
study, both IP and OP TMF tests have been performed in two crystal orientations, the $\langle 001\rangle$ and $\langle 011\rangle$ orientations respectively. The aim is not only to investigate the difference in TMF behaviour but also to study if there are any differences in deformation and damage mechanisms between the two loading conditions and crystal orientations. To study both the $\langle 001\rangle$ and $\langle 011\rangle$ orientations is of importance since crack propagation on the blade platform sometimes is dependent on properties in different crystal orientations, where the $\langle 011\rangle$ direction is one of the most important.

\section{Experimental}

The Ni-based single-crystal superalloy MD2 with chemical composition $\mathrm{Ni}, 5.1 \mathrm{Co}, 6.0 \mathrm{Ta}, 8.0 \mathrm{Cr}, 8.1 \mathrm{~W}, 5.0$ $\mathrm{Al}, 1.3 \mathrm{Ti}, 2.1 \mathrm{Mo}, 0.1 \mathrm{Hf}$ and $0.1 \mathrm{Si}$ in wt.\% was used for this study. The material was solution heat treated at $1290^{\circ} \mathrm{C}$ for $8 \mathrm{~h}$ before a two-stage ageing process with $3 \mathrm{~h}$ at $1100{ }^{\circ} \mathrm{C}$ and $24 \mathrm{~h}$ at $850^{\circ} \mathrm{C}$. Specimens oriented along their $\langle 001\rangle$ and $\langle 011\rangle$ crystallographic orientations with a diameter of approximately $6 \mathrm{~mm}$ were machined from cast bars. The deviation from the ideal crystal orientation was less than $10^{\circ}$ for all specimens. A servo-hydraulic TMF machine from Instron with induction heating and forced cooling was used for the TMF tests. Prior TMF testing, the machine was carefully aligned to prevent buckling of the specimens. A strain-controlled TMF cycle from $100-750^{\circ} \mathrm{C}$ was applied for all specimens with a temperature rate of $5^{\circ} \mathrm{C} / \mathrm{s}$. Figure 1 displays a simplified plot of the TMF cycles used in this study. For each TMF cycle, a hold time of 5 min was applied at $\mathrm{T}_{\max }$. The IP tests were performed with $\mathrm{R}_{\varepsilon}=0$ while for the OP tests $\mathrm{R}_{\varepsilon}=-\infty$. For the $\langle 001\rangle$ direction three different mechanical strain ranges $\left(\Delta \varepsilon_{\text {mech }}\right)$ were tested: $1.1,1.25$ and $1.4 \%$ respectively. For each $\Delta \varepsilon_{\text {mech }}$, one specimen was subjected to IP TMF cycling and another specimen to OP TMF cycling. Hence, altogether six $\langle 001\rangle$ oriented specimens were tested. However for the $\langle 011\rangle$ direction, only one $\Delta \varepsilon_{\text {mech }}$ was tested: $0.8 \%$. Both IP and OP TMF were studied also for the $\langle 011\rangle$ direction, hence for this direction only two specimens were tested. All specimens were cycled to failure where the number of cycles to failure $\left(\mathrm{N}_{\mathrm{f}}\right)$ was defined at $60 \%$ load drop.

After the tests, all specimens were examined by stereomicroscopy in order to investigate the fracture surfaces. Subsequently, the specimens were cut parallel to the loading direction for microstructural investigations by scanning electron microscopy (SEM). The SEM were prepared by grinding and mechanical polishing and no samples were etched. A Hitachi SU70 FEGSEM, with an annular electron backscatter detector making it possible to produce images by electron channelling contrast imaging (ECCI) with acceleration voltages from 10 to $20 \mathrm{kV}$, was used.

\section{Results and discussion}

\subsection{The TMF behaviour}

Figure 2 displays results from the TMF tests where the mechanical strain range, $\Delta \varepsilon_{\text {mech }}$, is plotted versus cycles

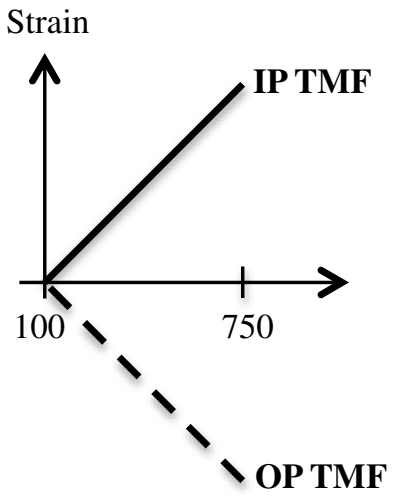

Figure 1. A simplified plot showing the strain-temperature cycle during the TMF tests performed in this study.

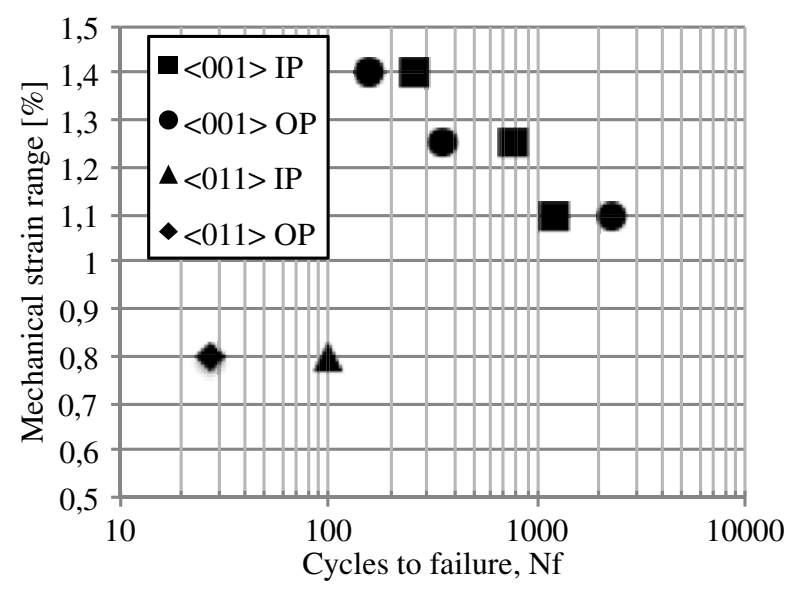

Figure 2. Results from the TMF tests; mechanical strain range, $\Delta \varepsilon_{\text {mech }}$, versus cycles to failure, $\mathrm{N}_{\mathrm{f}}$.

to failure, $\mathrm{N}_{\mathrm{f}}$. Due to its lower Young's modulus, the $\langle 001\rangle$ direction shows better fatigue properties during straincontrolled fatigue compared to the $\langle 011\rangle$ direction. In this case, better fatigue properties means a higher number of cycles to failure when equivalent strain ranges are compared. For the $\langle 001\rangle$ direction, it can be seen that for a high strain range $\left(\Delta \varepsilon_{\text {mech }}=1.4 \%\right)$, IP TMF cycling leads to a higher number of cycles to failure compared to $\mathrm{OP}$ cycling. For the medium strain range $\left(\Delta \varepsilon_{\text {mech }}=\right.$ $1.25 \%)$, IP still shows a longer fatigue life compared to OP. However, for the lower strain range $\left(\Delta \varepsilon_{\text {mech }}=1.1 \%\right)$ IP leads to a lower number of cycles to failure. Hence, the TMF life seems to be strain range dependent in this study. Regarding the $\langle 011\rangle$ direction where only one strain range was tested $\left(\Delta \varepsilon_{\text {mech }}=0.8 \%\right)$, IP cycling leads to a higher number of cycles to failure compared to OP cycling.

To further understand the TMF behaviour of the material it is important to study the hysteresis loops. Figures 3 and 4 displays the first two cycles from IP and OP TMF cycling at $\Delta \varepsilon_{\text {mech }}=1.4 \%$, respectively. Here, the typical hysteresis loops for IP and OP TMF cycling is visible. During IP TMF cycling, creep relaxation occurs in tension at the maximum temperature, while plastic deformation occurs in compression at the cold end of the TMF cycle, see Fig. 3. However, for OP TMF cycling, stress relaxation occurs in compression at the hot end of 


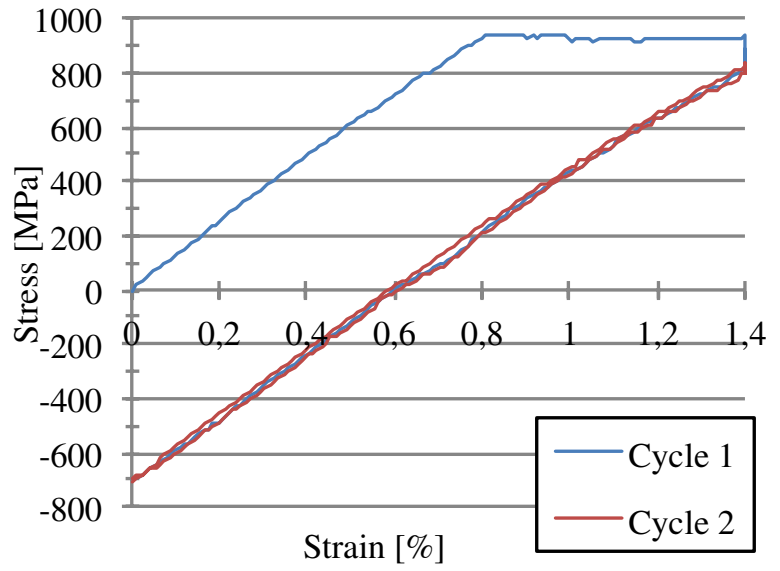

Figure 3. The stress-strain curve for the first 2 cycles of the $\langle 001\rangle$ oriented specimen subjected to IP TMF with $\Delta \varepsilon_{\text {mech }}=1.4 \%$.

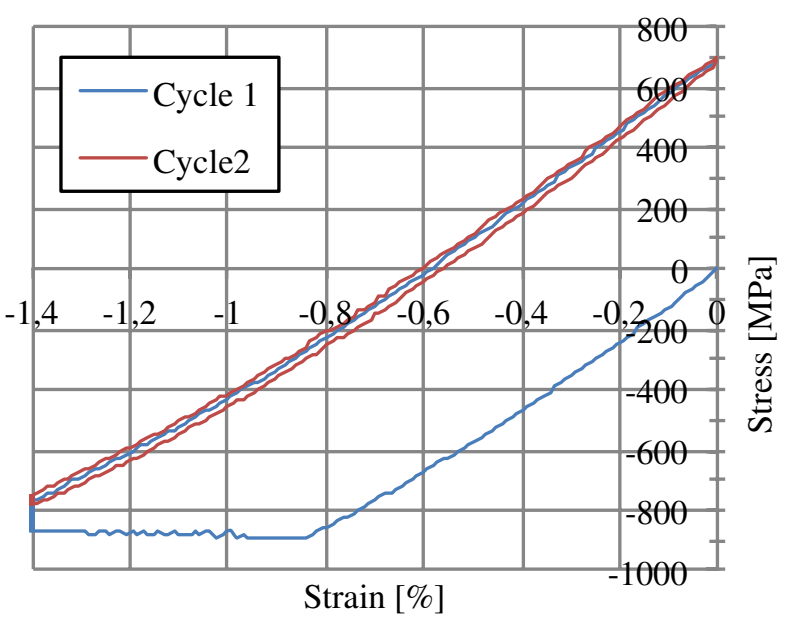

Figure 4. The stress-strain curve for the first 2 cycles of the $\langle 001\rangle$ oriented specimen subjected to OP TMF with $\Delta \varepsilon_{\text {mech }}=1.4 \%$.

the TMF cycle, while plastic deformation in tension is obtained at the cold end of the cycle, see Fig. 4.

A significant difference between the $\langle 001\rangle$ and $\langle 011\rangle$ directions is observed when loading into the first TMF cycle. For the $\langle 011\rangle$ direction, a serrated yielding is observed in both IP and OP. Figure 5 shows the two first cycles for the $\langle 011\rangle$ oriented specimen subjected to OP TMF. Here it is visible that a serrated yielding behaviour is present during the first cycle while the second cycle is more stable. However, for the $\langle 001\rangle$ direction the yielding is much more stable already in the first cycle for both IP and OP cycled material, Figs. 3-4. Hence, the serrated yielding behaviour when loading into the TMF cycle seems to be more extensive for the $\langle 011\rangle$ direction compared to the $\langle 001\rangle$ direction. In literature, a serrated yielding behaviour may sometimes be attributed to deformation twinning within the microstructure. However in this study, microstructural studies show that twinning does occur in both the $\langle 001\rangle$ and $\langle 011\rangle$ directions (please see Sect. 3.3 for a detailed discussion regarding the microstructure). Therefore, it is not believed that the serrated yielding occurs due to twinning in this case. Instead, previous studies $[10,11]$ have reported of a serrated yielding behaviour for the $\langle 011\rangle$ direction, and it was discussed that

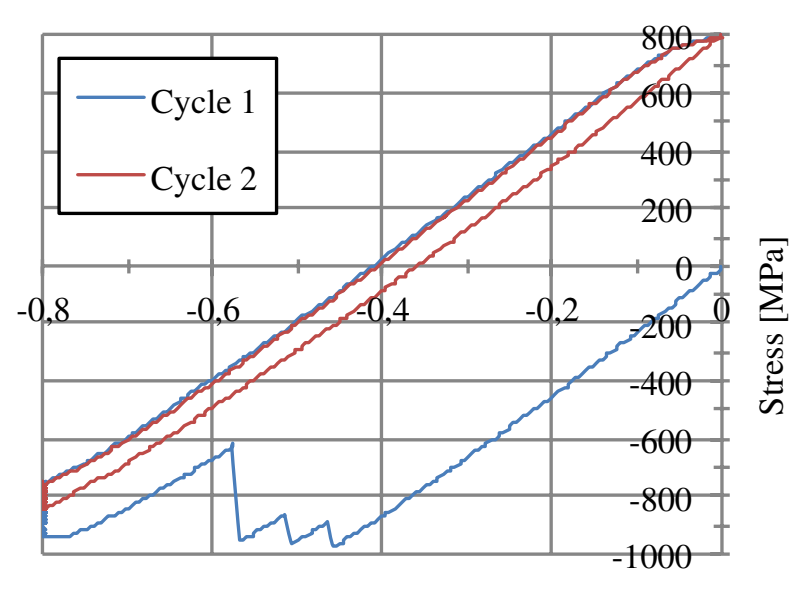

Strain [\%]

Figure 5. Serrated yielding during the first cycle of the $\langle 011\rangle$ oriented specimen subjected to OP TMF with $\Delta \varepsilon_{\text {mech }}=0.8 \%$.

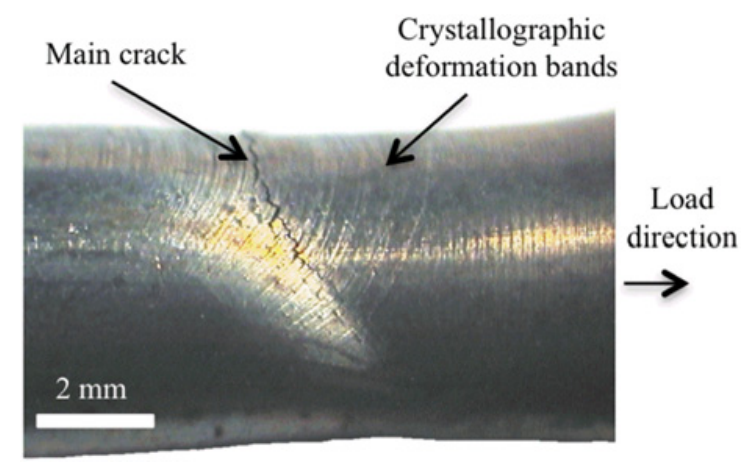

Figure 6. Surface of the $\langle 011\rangle$ oriented specimen subjected to IP TMF. Both the main crack and crystallographic deformation bands are visible on the specimen surface.

dynamic strain ageing has occurred and also that only one slip system is active during plastic deformation in the $\langle 011\rangle$ direction. If a serrated yielding behaviour during the first cycles will influence the TMF life has not been considered in this paper, but this may be of interest for future research.

\subsection{Fracture appearance}

The fracture appearance was studied by stereomicroscopy. For all specimens, the fractures seems to be partly crystallographic and partly random. The investigation shows that specimens subjected to OP TMF conditions have a higher tendency to show partly crystallographic fracture, while the specimens subjected to IP conditions have a tendency to show more random fractures. Moreover, crystallographic deformation bands are sometimes visible on the specimen surfaces. Here the $\langle 011\rangle$ specimens generally show more distinct crystallographic deformation bands compared to the $\langle 001\rangle$ oriented specimens. Hence, the $\langle 011\rangle$ direction shows both a serrated yielding behaviour and crystallographic deformation bands on the surface. On some specimens, deformation bands in different directions are observed; see Fig. 6 for such an example. Here the main crack has propagated along a deformation band going in a different orientation 


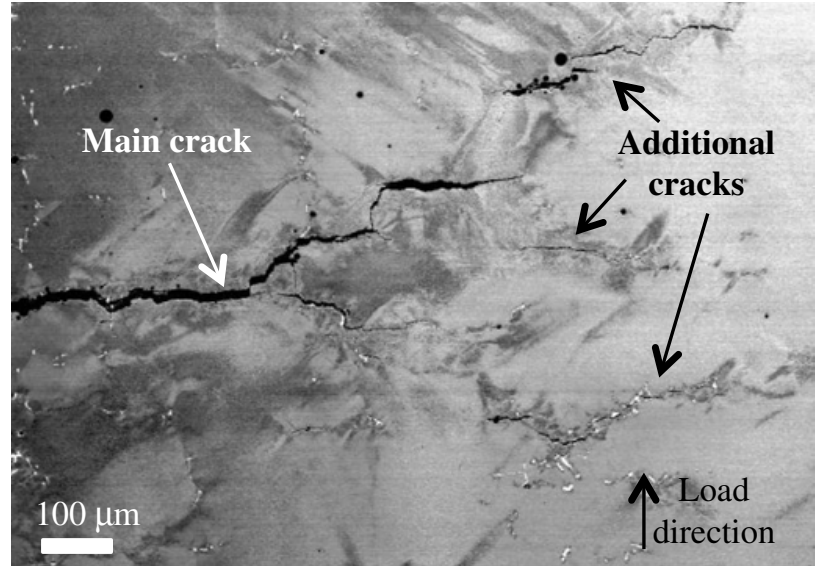

Figure 7. An ECCI image showing severe plastic deformation around both the main crack as well as some additional cracks for the $\langle 001\rangle$ IP specimen subjected to $\Delta \varepsilon_{\text {mech }}=1.1 \%$.

compared to other deformation bands. However, all deformation bands are consistent with slip along one of the $\{111\}$ crystallographic planes.

\subsection{Scanning electron microscopy}

When studying the specimens by SEM, no rafting (directional coarsening of the $\gamma^{\prime}$-precipitates) of the microstructure is observed. It seems that $750{ }^{\circ} \mathrm{C}$ is a too low temperature to initiate the rafting phenomena during TMF cycling in this case. From the SEM investigation of the $\langle 001\rangle$ oriented specimens, a difference between IP and OP TMF cycling is the appearance of cracks within the microstructure. For the $\langle 001\rangle$ IP specimens, several smaller cracks are observed in addition to the main crack. Figure 7 displays such an appearance where the main crack not only is surrounded by severe plastic deformation but also several additional cracks. The smaller cracks seem to have initiated around pores and other defects. On the other hand, in $\langle 001\rangle$ oriented specimens subjected to OP TMF cycling less smaller cracks are observe in addition to the main crack.

Deformation twinning within in the $\gamma / \gamma^{\prime}$-microstructure is found in all specimens: in both the $\langle 001\rangle$ and $\langle 011\rangle$ directions as well as from both IP and OP conditions. However for the $\langle 001\rangle$ direction, the twinning phenomena seems to be much more pronounced in the specimens subjected to OP TMF cycling, see Fig. 8. In the $\langle 001\rangle$ IP specimens, the microstructure instead is characterised by a large amount of additional cracks and less twins. According to Zhang et al. [12,13] and Sato et al. [13] twins are created in compression for $\langle 001\rangle$ oriented specimens when the number of TMF cycles is relatively low. In Ref. [12] it was also stated that twins arise in the $\gamma$-phase and afterwards propagates through the $\gamma / \gamma^{\prime}$-microstructure by partial dislocation glide along an $\{111\}$ plane. In addition, Kovarik et al. [8] discuss that deformation twinning occurs at temperatures around $700{ }^{\circ} \mathrm{C}$. This may explain why the twinning is much more pronounced during OP TMF, where the compressive stresses are obtained at high temperature, compared to IP

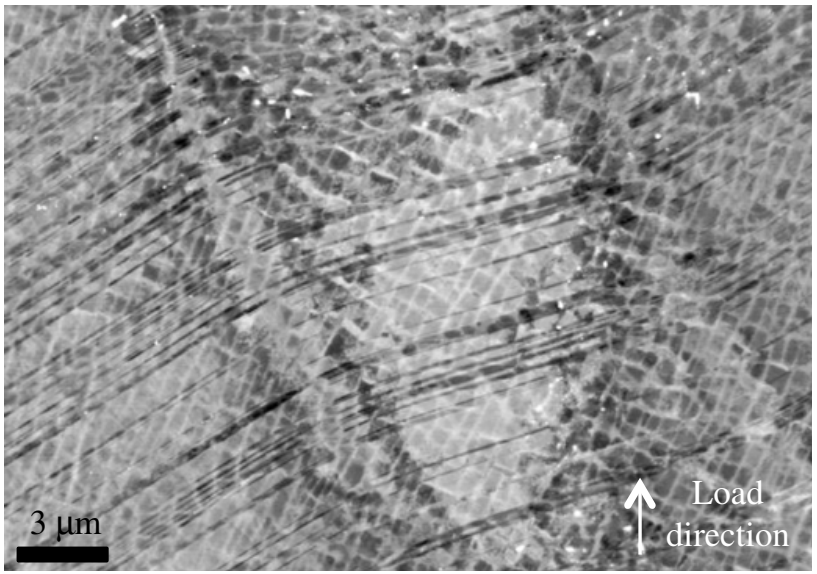

Figure 8. An ECCI image showing extensive twinning in the $\langle 001\rangle$ OP specimen subjected to $\Delta \varepsilon_{\text {mech }}=1.4 \%$.

TMF, where the compressive stresses are obtained at low temperature.

For the $\langle 011\rangle$ direction the opposite behaviour is observed; here the twinning is more pronounced in the specimen subjected to IP TMF compared to OP. In fact, the microstructures of the $\langle 011\rangle$ IP specimens are very similar to the $\langle 001\rangle$ OP specimens. A previous study [6] has shown that there might be a change in deformation mechanism for the $\langle 011\rangle$ direction when going from low to high strain ranges during OP TMF. In that study, specimens subjected to low strain ranges showed twinning, while for specimens subjected to a higher strain ranges, the deformation mechanism was rather localised bands with a sheared of the $\gamma / \gamma^{\prime}$-microstructure. Since the mechanical strain range in this study is rather high $(0.8 \%)$, the result that no twinning has occurred in the $\langle 011\rangle$ OP specimen, seems to agree well with the previous study. From this, one may conclude that a low mechanical strain range is needed to obtain twinning in the $\langle 011\rangle$ direction during OP TMF.

All twins have not always propagated in the same direction. Instead, it is quite common that twins have propagated in different directions; see Fig. 9 for such appearance. At the intersection of twins propagating in different directions, precipitation of topologically closepacked (TCP) phases often occurs. The TCP phases appear as bright spots in the electron backscattered images and are probably $\mu$-phase (Fig. 9). From this figure, it also seems that the precipitation of TCP phases is preferred to the $\gamma$-channels rather than in the $\gamma^{\prime}$-cuboids. The reason for this behaviour is not yet understood and has to be further investigated.

The size of the twins varies and sometimes thinner twins are created within thicker twins, see Fig. 10 for such an example. In that image one can see that the largest twin contains at least five smaller twins, which are all of nano size. Once again it is visible that precipitation of TCP phases has occurred at the twin intersections and in Fig. 10 it is also visible that recrystallization has occurred at these twin intersections. Figure 11 displays another example where recrystallization and TCP formation have occurred at twin intersections. In this image at least five recrystallized grains have nucleated at the twin 


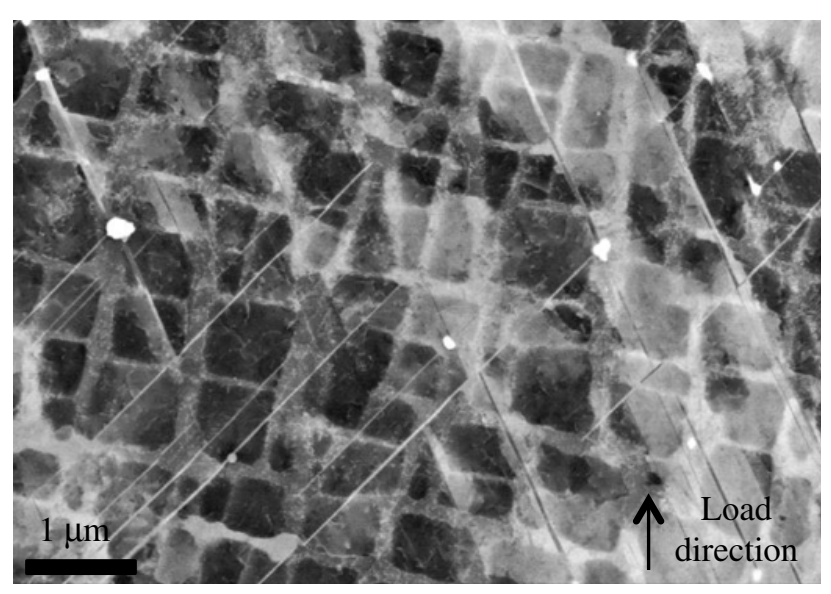

Figure 9. An ECCI image showing how twins have propagated in different directions in the $\langle 001\rangle$ OP specimen subjected to $\Delta \varepsilon_{\text {mech }}=1.1 \%$.

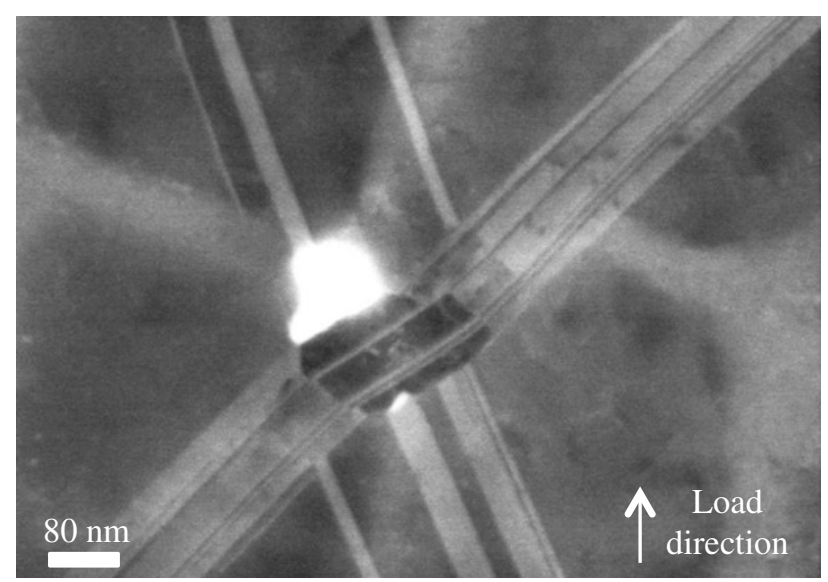

Figure 10. An ECCI image showing how TCP formation and recrystallization have occurred at a twin intersection in the $\langle 001\rangle$ OP specimen subjected to $\Delta \varepsilon_{\text {mech }}=1.1 \%$. In addition, twins seem to have grown inside another twin.

intersections. To trigger recrystallization, a great amount of plastic strain has to be induced before the material is subjected to high temperatures. However, recrystallization at a relatively low temperature as $750{ }^{\circ} \mathrm{C}$ is still not well reported in the literature. From this study one may assume that the intersection of twins induces enough plastic strain and that $750{ }^{\circ} \mathrm{C}$ is a sufficient temperature to initiate recrystallization during TMF cycling.

Further investigation shows that the intersection of twins, where TCP formation and recrystallization have occurred, seems to be an initiation point for damage within the material. Figure 12 displays two backscattered electron images where (b) is a magnification of (a). In Fig. 12a, a crack at the specimen surface is observed and the crack has grown in a crystallographic way along twin plates. From this image one can also see that the deformation is crystallographic to a great extent. Further, in Fig. 12b severe plastic deformation parallel to where the crack has initiated is clearly visible. The image displays extensive twinning within the $\gamma / \gamma^{\prime}$-microstructure where the twin

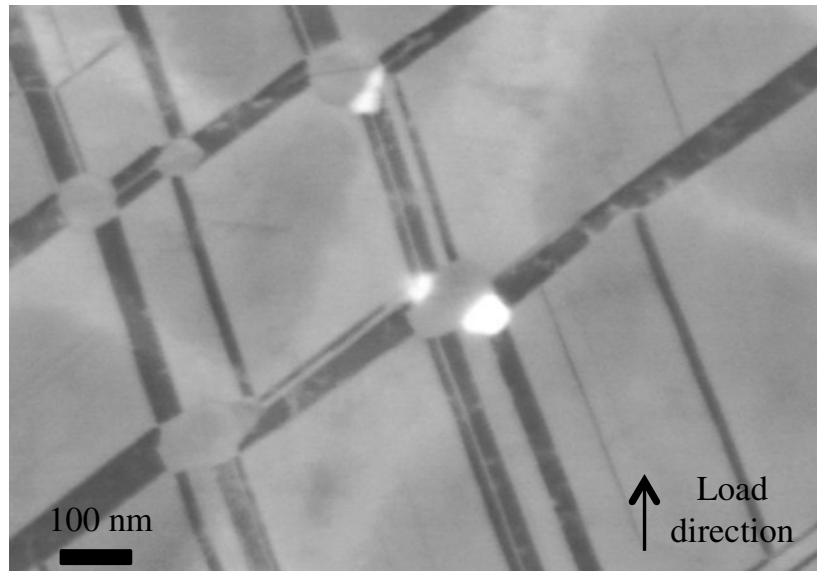

Figure 11. An ECCI image showing how TCP formation and recrystallization have occurred at twin intersections in the $\langle 001\rangle$ OP specimen subjected to $\Delta \varepsilon_{\text {mech }}=1.1 \%$.
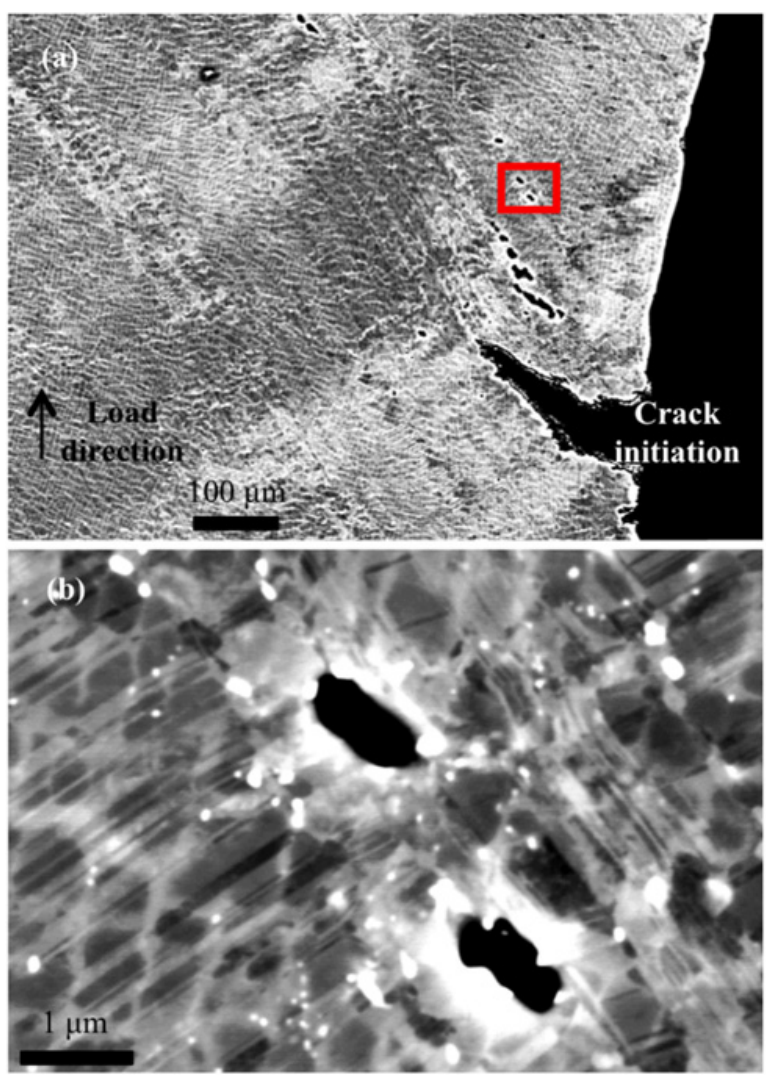

Figure 12. A backscattered electron image showing the $\langle 011\rangle$ IP specimen subjected to $\Delta \varepsilon_{\text {mech }}=0.8 \%$, (a) crack initiation along twin plates, (b) magnification of (a) where damage is visible at twin intersections.

plates have grown in different orientations. It seems that the intersection of propagating twins have triggered TCP formation, recrystallization and finally a damage within the material (the black voids). Hence, the voids are located along twin plates and it is probable that those voids together with the twin plates create a preferential path for crack propagation. Since superalloys in single-crystal form do not have any grain boundary strengthening alloying 
elements such as carbon or boron, recrystallized areas can be considered as weak areas and therefore be assumed as crack initiation points during TMF cycling. Hence is it of great importance to understand the recrystallization process in order to improve the TMF properties of a single-crystal superalloy. A previous study [5] has stated that twinning is a major deformation mechanism during TMF and also that the intersection of twins might trigger recrystallization. The same study also found that crack propagation is a consequence from recrystallization and not the other way around, this statement was also confirmed by Segersäll et al. [6]. The present study once again show indications that the recrystallization phenomena occurs prior crack propagation and not the other way around.

Since twinning seems to be the major deformation mechanism during TMF cycling, it is of great importance to study this phenomena in order to understand the TMF behaviour. According to Kovarik et al. [8,9] microtwinning occurs at intermediate temperatures, approximately 660$700{ }^{\circ} \mathrm{C}$, and re-ordering within the $\mathrm{L}_{2}$-ordered $\gamma^{\prime}$-phase is believed to be the rate controlling process. The present study has strengthened the fact that twinning is major deformation mechanism in single-crystal superalloys during TMF. However, further studies are needed in order to fully understand the formation of twins and their influence on TMF properties.

\section{Conclusions}

From TMF testing at $100-750{ }^{\circ} \mathrm{C}$ of the Ni-based singlecrystal superalloy MD2 the following conclusions can be drawn:

- IP and OP TMF cycling results in different fatigue lives where IP leads to longer life at high strain range while OP cycling leads to longer fatigue life at a lower strain range.

- No significant differences in deformation and damage mechanisms were found between the strain ranges that led to different fatigue lives.

- A clear difference between IP an OP was found: for the $\langle 001\rangle$ direction twinning seems to be much more pronounced during OP TMF while for the <011〉 direction IP TMF cycling leads to twinning.
- The intersection of twins seems to trigger precipitation of TCP phases as well as recrystallization. Those areas seem to be weak points where crack initiation and propagation is preferred.

The work has been financially supported by Siemens Industrial Turbomachinery $\mathrm{AB}$ in Finspång, Sweden and the Swedish Energy Agency, via the Research Consortium of Materials Technology for Thermal Energy Processes, Grant No. KME-502. In addition, the support from the Swedish Government Strategic Research Area in Materials Science on Functional Materials at Linköping University (Faculty Grant SFO-Mat-LiU \# 200900971) is also acknowledged.

\section{References}

[1] R. C. Reed, The Superalloys - Fundamentals and Applications (Cambridge University Press, Cambridge, 2006)

[2] J.J. Moverare, M. Segersäll, A. Sato, S. Johansson, R. Reed, Superalloys 2012, ed. E. S. Huron et al., 369 (2012)

[3] G.M. Han, J.J. Yu, X.F. Sun, Z.Q. Hu, Mater. Sci. Eng. A, 528, 6217 (2011)

[4] F. Liu, Z.G. Wang, S.H. Ai, Y.C. Wang, X.F. Sun, T. Jin, H.R. Guan, Scr. Mater., 48, 1265 (2003)

[5] J.J. Moverare, S. Johansson, R.C. Reed, Acta Mater., 57, 2266 (2009)

[6] M. Segersäll, J.J. Moverare, K. Simonsson, S. Johansson, Superalloys 2012, ed. E. S. Huron et al., 215, (2012)

[7] H.U. Hong, J.G. Yoon, B.G. Choi, I.S. Kim, Y.S. Yoo, C.Y. Jo, Int. J. Fatigue, DOI:10.1016/ j.ijfatigue.2013.01.015 (2013)

[8] L. Kovarik, J. Unocic, J. Li, M.J. Mills, JOM, 61, 42 (2009)

[9] L. Kovarik, J. Unocic, J. Li, P. Sarosi, C. Shen, Y. Wang, M.J. Mills, Prog. Mater. Sci., 54, 839 (2009)

[10] M. Segersäll, J.J. Moverare, Materials, 6, 437 (2013)

[11] M. Segersäll, J.J. Moverare, D. Leidermark, K. Simonsson, Metall. Mater. Trans. A, DOI:10.1007/s11661-014-2198-0 (2014)

[12] J.X. Zhang, H. Harada, Y. Ro, Y. Koizumi, T. Kobayashi, Acta Mater, 56, 2975 (2008)

[13] A. Sato, J.J. Moverare, M. Hasselqvist, R.C. Reed, Metall. Mater. Trans. A, 43A, 2302 (2012) 